\title{
Los discursos de las garzonas en las salas de cerveza del norte de Chile. Género y discriminación*
}

\author{
Paulina Salinas \\ Universidad Católica del Norte, Antofagasta, Chile. Email: psalinas@ucn.cl \\ Jaime Barrientos \\ Universidad Católica del Norte, Antofagasta, Chile
}

\begin{abstract}
Resumen: Este trabajo analiza los discursos de las mujeres/garzonas, que trabajan en las salas de cerveza (schoperías) sobre la discriminación de género y los modelos relacionales que se establecen en estos espacios entre clientes y garzonas. Se postula que estos aspectos recién mencionados, coadyuvan a la permanencia de una identidad masculina hegemónica, que se intensifica en el contexto de la minería. Los antecedentes obtenidos muestran que las garzonas- en su mayoría mujeres oriundas de los países limítrofes y/o del sur de Chile-, se encuentran afectadas por una situación de vulnerabilidad social y económica, precariedad laboral y, además, cargan con una serie de imaginarios sociales en torno a ellas, a los lugares en los que trabajan (schoperías) y a la ciudad en la que habitan. Es decir, son mujeres discriminadas. Estas trabajadoras se encuentran atrapadas entre el proceso de crecimiento económico y atracción que ofrece la industria minera en el norte del país y, a la vez, a las expectativas que tienen por mejorar su situación familiar. Asimismo, se ven confrontadas por la fuerza de un sistema sociocultural de sexo/género hegemónico que favorece relaciones de poder asimétricas y la subordinación femenina, lo que contribuye a reforzar identidades de género y modelos relacionales de inequidad, que son naturalizados en este entorno productivo.
\end{abstract}

Palabras clave: discursos, relaciones de género, discriminación, minería.

\section{The speeches of the waiters in the beer halls in Northern Chile. Gender and discrimination}

\begin{abstract}
This paper aims to identify the presence of gender discrimination and examine existing relational models of women working in beer halls (shoperías) in northern Chile. Through discourse analysis, it will show how these societal constructs contribute to hegemonic masculine identity, which is further intensified by the mining context which is prevalent in the region. Data shows that female servers, who are mainly from the south of Chile or neighboring countries, are (largely, somewhat, mildly) affected by stereotypes concerning appearance and behavior that lead to social vulnerability, as well as job instability that affects their economic security. These workers are attracted by opportunities due to economic growth and are influenced by the expectation to improve their family situation. Female servers in the schoperías work in a sociocultural system of sex and gender relations which favors asymmetrical hegemonic power, resulting in female subordination and relational models of inequity that are normalized in their
\end{abstract}


immediate work environment.

Key words: discourses, gender relations, discrimination, mining industry.

\section{Os discursos dos garçons nos salões de cerveja Norte do Chile. Discriminação e gênero}

Resumo: Este artigo analisa os discursos das mulheres / garçons que trabalham em salas de cerveja (schoperías) sobre a discriminação de gênero e os modelos relacionais estabelecidas nesses espaços entre os clientes e garçons. Nossa hipótese é que esses problemas já mencionados, contribuem para a persistência de uma identidade masculina hegemônica, que se intensifica no contexto da mineração. Os dados obtidos mostram que os garçons, a maioria mulheres indígenas de países vizinhos e / ou para o sul do Chile, são afetadas por uma situação de vulnerabilidade social e econômica, precariedade do emprego, e também carregam uma série de imaginários sociais em torno deles, os lugares onde trabalham (schoperías) ea cidade onde vivem. Ou seja, são mulheres discriminadas. Estes trabalhadores estão presos entre o processo de crescimento econômico e atração que oferece a indústria de mineração no norte e, ao mesmo tempo, as expectativas que eles têm de melhorar a sua situação familiar. Além disso, são confrontadas pela força de um sistema cultural de sexo / gênero hegemônico favorecendo relações assimétricas de poder e subordinação das mulheres, o que contribui para o reforço das identidades de gênero e os modelos relacionais de desigualdade que são naturalizados neste ambiente produtivo.

Palavras-chave: discurso, gênero, discriminação, exploração mineira.

\section{Introducción}

La igualdad de oportunidades y la no discriminación han sido objetivos transversales de los gobiernos en Chile desde la recuperación de la democracia (1990), sin embargo, aunque han sido muchos los logros alcanzados, sin duda mejorar las condiciones económicas, sociales y laborales de las mujeres, constituye aun, un desafío pendiente. En esta dirección, existen diversos estudios que analizan la situación de desigualdad que ha afectado a las mujeres en lo económico, político, social y laboral (Valdés y Weinstein, 1993; Ruiz, y otros, 1998; Valdés, 2001; GIM, 2002; Lamas, 2002; Salinas 2002, Gutiérrez, 2007, Carbonero, 2007). Específicamente, la participación laboral es central, por su relación con los grados de autonomía que alcanzan los sujetos; así también, es relevante en la realización personal, calidad de vida de la familia y la superación de la pobreza.

$\mathrm{Al}$ respecto, los antecedentes existentes registran un crecimiento sostenido de la participación femenina en el mercado del trabajo en América Latina, llegando inclusive a tasas superiores a la de los hombres (OIT, 2007). No obstante, también se ha incrementado la segmentación ocupacional, pues son las mujeres las que se concentran en los empleos más precarios. A pesar que el nivel de escolaridad ha aumentado, pasando de 8 a 9 
años, y que el porcentaje de ellas en ocupaciones profesionales y técnicas ha crecido más del 50\% (Abramo, 2004), continúan existiendo mecanismos de discriminación ocupacionales que las limitan a las áreas menos valorizadas del mercado.

En Chile, aunque la participación laboral está aumentando, sigue siendo la más baja de la región, con brechas importantes de más de 10 puntos porcentuales respecto de países de similar nivel de desarrollo socio económico como Argentina (49\%) y Uruguay (51,9\%) (CEPAL, 2008). Según cifras de la Encla (2009), a nivel nacional la tasa de participación femenina es de $32,7 \%$ y la de los hombres es de un $67,3 \%$.

Por otra parte, aunque existe una mayor escolaridad por parte de las mujeres frente a los hombres, 11,8 años y 10,7 años respectivamente (OIT, 2007), éstas, de igual forma, obtienen menores ingresos. De acuerdo a las cifras disponibles, el 2005, las trabajadoras ganaban, en promedio, el 79\% de lo que percibían ellos. La brecha de ingresos aumenta con el nivel de educación, es decir, cuando las mujeres tienen estudios universitarios su salario, en promedio, sólo representa el $66 \%$ del sector masculino (INE, 2007).

Esta situación se reproduce, a escala regional, pues en Antofagasta, como zona eminentemente minera (minería del Cobre), la participación laboral de las mujeres es menor, y se concentra fuertemente en el área de servicios y comercio (38,3\%, y 35,9\% respectivamente, en el año 2004). Aunque, la minería genere altos ingresos, ésta tiene efectos en la demanda por servicios y bienes, activando diversas ramas del sector terciario. Y, este último, tiene menos barreras de género, pero circunscribe a las mujeres a desempeñar roles entendidos como tradicionalmente femeninos (SERNAM, 2009).

Además, la creciente incorporación de tecnología a los procesos productivos, asociado a los altos estándares de modernización de la gran minería del cobre en Chile, no ha significado una mayor presencia de las mujeres en estos mercados; por el contrario su participación es aún marginal, debido a la conocida segregación por sexo de este sector laboral.

Por lo tanto, el objetivo de este estudio ha sido a través de una investigación cualitativa profundizar en la situación que experimentan las trabajadoras, mujeres garzonas, que se desempeñan en las salas de cerveza o schoperías de Calama, ya que como los principales lugares de esparcimiento de los trabajadores mineros en la región, éstos son sitios privilegiados para la reproducción de la discriminación de género y el establecimiento de modelos relacionales específicos entre clientes y garzonas, que coadyuvan en la vigencia de una identidad masculina hegemónica, intensificada en este sector productivo. Por lo mismo, se puso el énfasis en analizar los discursos de las mujeres como protagonistas de la relación que se produce entre mujer-trabajo y minería. 


\section{Antecedentes teóricos}

En el marco de la situación laboral que experimentan las mujeres en la II Región, nos interesa analizar los fenómenos asociados a esta realidad, como identidad, discriminación, y masculinidad.

La identidad de género de las mujeres ha estado, históricamente, relacionada a un largo proceso de discriminación en los distintos ámbitos de la vida social, lo que ha sido un factor determinante en las diferencias y relaciones inequitativas entre hombres y mujeres. La discriminación, a la vez, ha mantenido, por una parte, la desvalorización respecto a los beneficios de carácter social y económico que se obtienen del trabajo de la mujer, y por otra, la visión que se tiene de este sector como sujetos no productivos. Además, esta construcción ha amparado diferencias con respecto al sexo, roles, posiciones y condiciones entre los géneros (Quintero, 2007). Por lo tanto, la discriminación como proceso de diferenciación desigual, condiciona la construcción del género, la identidad y los modelos relacionales, y no representa sólo un conjunto de características o roles, sino es el producto de ciertas prácticas sociales que se desarrollan a través de la interacción de los individuos (West y Zimmerman, 1999).

Trautner (2005) plantea que las identidades también tienen un carácter colectivo, que inclusive traspasa los ámbitos organizacionales, es decir, hay órdenes simbólicos de género que modelan a los sujetos, en términos de cómo comportarse en determinados contextos sociales. Otras investigaciones han abordado las relaciones de género en las sociedades latinoamericanas y la construcción de la identidad masculina (Valdés y Olavarria, 1997, Viveros, 2001; Careaga y Cruz, 2006; Olavarría, 2006; Seidler, 2006). Estos estudios, inspirados en las investigaciones sobre mujeres, han puesto atención en el carácter construido y multifacético de la identidad. Connell $(2003,2006)$ afirma que los modelos de masculinidad cambian con el tiempo, en una misma sociedad, inclusive en una comunidad o institución (Valdés y Olavarría, 1997). En el caso de Chile, Valdés y Olavarría (1997) y Olavarría (2006) coinciden en que no existe una única identidad masculina, sino que más bien se trata de un proceso complejo, a pesar del impacto que han tenido los modelos hegemónicos de masculinidad existentes. Aunque, como lo han señalado Connell (2003) y Seidler (2006), la identidad masculina hegemónica se intensifica en los espacios de homosociabilidad, como clubes deportivos, bares, cafés y salas de cerveza (shoperías), entre otros. En estos lugares de interacción se refuerzan los estereotipos de género asociados tanto a los hombres como a las mujeres que allí trabajan.

Sin embargo, hay algunas investigaciones que se aventuran sobre el actual proceso de transición que están experimentando los varones en la construcción de su identidad (Carabi y Armengol, 2008; Montesino, 2007, Salinas, 2007, Salinas \& Arancibia, 2006, Valcuende y Blanco, 2003). Esta transición ha implicado un cuestionamiento a los estereotipos tradicionales asignados a los hombres y mujeres. Y, aunque estas transformaciones no 
representan una realidad generalizada, dan cuenta de modificaciones en los imaginarios colectivos y marcan nuevas pautas de interacción en la vida cotidiana (Martinez, 2007), lo que estaría impactando positivamente en la vida social y en las relaciones de género.

Estos antecedentes son relevantes para comprender las particularidades que estos fenómenos tienen en el contexto de la minería, ya que las investigaciones existentes, reportan el carácter masculino del movimiento obrero y sindical, de los partidos políticos y de la organización laboral (Aylwin y otros, 1998; Salazar y Pinto, 1999; Zapata, 2002; Vergara, 2004; Carmagnani, 2006). También, estudios psicosociales analizan la situación de los hombres trabajadores, de las mujeres, las condiciones familiares, los efectos del sistema de turno y los roles que los sujetos desempeñan en la vida privada de los campamentos en las minas (Vergara, 2007; Montecino, 1998). Thomas Klubock (1995), en un importante estudio sobre el mineral El Teniente, en la zona centro sur del país, destaca la relevancia de la ideología de género en el modelamiento de la clase trabajadora y resalta cómo se estructuraron las identidades de la clase y de la comunidad que surgieron en el proceso de proletarización. Este autor afirma que son diversas las fuerzas que se combinaron para producir la masculinización del trabajo y la domesticación de las mujeres en la esfera privada. El matrimonio, como sustento de la producción minera, proporcionó un orden y una disciplina laboral que construyó una identidad totalmente masculinizada. Sagredo y Gazmuri (2005), coincidiendo con lo anterior, afirman que el mundo del cobre era de los hombres y la vida de la mujer estaba restringida a las funciones del hogar y al cuidado de los hijos.

Otro estudio sobre los discursos del minero como representaciones de un sistema social y un estilo de vida determinado, evidenciaron contradicciones y tensiones las que intentan ocultar que los hombres estarían experimentando en alguna medida un proceso de reciclaje de su masculinidad (...) como una forma de mantener el status quo (Salinas y Arancibia, 2006). Luego, en una etnografía realizada en los locales de venta de cerveza frecuentados por trabajadores mineros en Calama, en la Región de Antofagasta se constató la vigencia que tiene en estos espacios de encuentro de hombres la performance de la masculinidad hegemónica (Barrientos y otros, 2009). Precisamente este primer acercamiento nos permitió acceder al universo simbólico y relacional de estos espacios, emergiendo las mujeres como ejes centrales en el negocio de la diversión y el esparcimiento masculino.

Por lo tanto, todas estas investigaciones van evidenciando la intensidad de la dominación masculina y, como lo plantea Bourdieu (2007), la importancia que tiene el discurso en materia de violencia simbólica y de cómo ésta es capaz de generar una segregación entre el mundo femenino y masculino, instalándolas a ellas en espacios totalmente diferentes.

La violencia simbólica se instaura como un mecanismo invisible, amparado bajo la impresión reductora de su carácter simbólico, haciendo que en el colectivo se crea que no traerá consecuencias reales. Por tanto, 
las estructuras de dominación masculina son producto del continuo trabajo de reproducción donde confluyen diversos agentes, ya sea el hombre como generador de violencia física y simbólica, como también instituciones entre las que sobresalen la familia, la escuela, la Iglesia y el Estado. De esta forma, se perpetúan concepciones sobre la imagen y posición de la mujer a través de un continuo habitus, hasta llegar así, a comprender como "natural” la supremacía del varón (Ibid: 2007)

\section{Minería, trabajo e identidad de género}

Actualmente Chile es el principal exportador mundial de cobre, en Antofagasta se encuentran los yacimientos más importantes del mundo. Entre 1999-2000, la producción de este mineral en la Segunda Región representó un $45 \%$, de las exportaciones de la nación y un $84 \%$ de la producción minera chilena. Por esto mismo la industria minera concentra un 95\% de las exportaciones y un 60\% del PIB de la región (Cademartori, 2009).

La Región de Antofagasta, ubicada en el extremo norte del país, tiene un total de 521.682 habitantes (CASEN, 2006). De acuerdo a las cifras que entrega INNOVA (2008), la Región contribuye con un 7,8\% al Producto Interno Bruto Nacional (PIB), siendo el cuarto aporte más alto de Chile, con aproximadamente 5.000 millones de dólares. Antofagasta se caracteriza por su dinamismo, entre 1990 y 2004 ha tenido la tasa más alta de variación media anual de crecimiento regional (6,9\%) (Ramírez y Silva, 2008).

El Norte de Chile, en especial la Región de Antofagasta, ha sido históricamente productor de gran parte del presupuesto del país y esta última década, sostenedor de la economía chilena. Es así como en el 2008 aportó con un 8\% al Producto Interno Bruto Nacional, y a nivel regional se plantea como la actividad productiva que entrega más de la mitad del ingreso per capita de Antofagasta, con un 64\% (Lardé, et al; 2008).

La minería es importante también por los recursos fiscales que reporta estos van mucho más allá de los ingresos que genera CODELCO, la empresa estatal más grande en el rubro. Ya que todas las empresas privadas pagan sus impuestos, en tasas variables dependiendo de la naturaleza de sus contratos de inversión. De hecho, la mayoría de las empresas mineras de gran escala están sujetas al régimen de tributación general que afecta con el impuesto de primera categoría y global complementario a las distribuciones de utilidades efectuadas a los propietarios, socios o accionistas. (Compendio de la minería chilena, 2009).

En la actualidad, el desarrollo de nuevos proyectos mineros, la renovación de la dotación en las faenas de más larga data y la utilización de alta tecnología en las actividades productivas, han generado la necesidad de incorporar un importante número de personas jóvenes al sector, cuya formación académica es creciente, dando origen a un nuevo perfil de trabajador, que se caracteriza por su capacidad técnica, polifuncionalidad y nivel de autonomía en la toma de decisiones y en el cumplimiento de nor- 
mas y procedimientos. El promedio de años de estudios de este empleado (minero) en el 2002 fue de 14 años. La mayor formación de este trabajador facilita su capacitación, entrenamiento y desarrollo de nuevas competencias profesionales (Consejo Minero, 2003).

En la minería, el trabajo es un mecanismo de realización para los hombres, a través de éste, forman un hogar, son proveedores y cumplen los roles socioculturales esperados para ellos (Ruitenbeek, 1967; Montesinos, 2002; Olavarría, 2000; Seidler, 2006). Los estudios coinciden en que los varones se encuentran exigidos por una dinámica en la que trabajo-dignidad y capacidad se relacionan en un círculo vicioso, que condiciona la idealización de la identidad masculina adulta (Ruitenbeek, 1967; Dunbar, 1994; Macheke y Campbell 1998; Salinas y Arancibia, 2006; Salinas, 2007). Las circunstancias laborales, los lugares de trabajo, las condiciones económicas y las estructuras de las organizaciones, influyen en la forma en cómo los hombres construyen la identidad (Connell, 2003). La dominación ejercida por ellos se manifiesta en la discriminación que se ejerce contra las mujeres, y se relaciona con el proceso de construcción de su propia identidad. Se expresa con una actitud activa, abierta o implícita, que la sociedad ayuda a controlar (Cortés, 2004). Así en la minería, se recrean verdaderos rituales de hombría, a través de un lenguaje soez, bromas y rumores (Barrientos y otros, 2009). Los hombres tienden a sexualizar los discursos, es decir, expresan sapiencia de los significados sexuales. Esto se observa, igualmente, en las interacciones entre clientes y garzonas en la schoperías (Barrientos y otros, 2009), ya que los hombres tienden a explicar que estos lugares son sitios de distracción.

Lo anterior justifica la ubicación de las mujeres en un lugar subordinado del sistema social y, la sexualización de las relaciones de trabajo, expresa las expectativa de que las mujeres deberían actuar utilizando la seducción, coquetería y femineidad para relacionarse con los hombres (Tallichet, 1995). Por lo tanto, en la minería hay una práctica fuertemente generizada; esto implica que la jerarquía social entre hombres y mujeres se encuentra legitimada y reforzada en la cultura organizacional. En estos espacios dominados por los hombres, sus privilegios se manifiestan a través de una diferenciación funcional del trabajo por género (Tallichet, 1995).

\section{Modelos relacionales y discursos de género en el contexto minero}

Las características particulares de la minería como actividad productiva tienen un impacto importante en la configuración de la identidad, en las relaciones entre los sujetos y en los discursos que allí se recrean.

Las relaciones de género, desde una perspectiva interaccionista, son vistas como procesos de relación mediante los cuales "los sujetos crean su propio mundo". Distintos autores coinciden en que cada situación de la interacción humana es específica y debe ser estudiada como un universo en sí mismo (Joas, 1995; Reynoso, 1998; Sandín, 2003; Andreú y otros, 2007). Además, en esta dinámica la gente "crea” el significado y el orden social 
mediante una especie de negociación. Erving Goffman (citado en Joseph, 1999) realza la relevancia que tiene la práctica social, no en sus motivos ni en su elaboración subjetiva, sino en sus consecuencias y vocabulario. Goffman afirma que un motivo no es la fuente subjetiva de la acción, sino un acto de lenguaje que se inscribe en un discurso disponible, para los actores sociales y la interpretación de su conducta.

Los interaccionistas señalan que la gente no se entiende directamente con la realidad, sino que la mediatiza y la interpreta a través de símbolos (lenguaje). En otras palabras, los seres humanos no reaccionan simplemente a una serie de estímulos o a las acciones de los demás, sino que la interpretan otorgándole significados (Reynoso, 1998). Estos significados se expresan en el discurso de los sujetos y éste es una unidad lingüística formada por una sucesión de oraciones (Maigret, 2003; Otero, 2006; West y Turner, 2004). Por lo tanto, en el discurso, la categoría de género es entendida como una construcción sociocultural que modula las relaciones y las prácticas de hombres y mujeres (Bonder, 1999:35), constituyéndose esta categoría en un organizador social primario que tiene una influencia central en la producción del mismo.

El objetivo de este trabajo, como ya lo mencionamos, es analizar desde una perspectiva cualitativa, la situación que experimentan las mujeres garzonas, desde la visión de ellas como protagonistas, que trabajan en las salas de cerveza o schoperías de Calama. Específicamente se explora la discriminación de género y el establecimiento de modelos relacionales, que contribuyen a mantener una identidad masculina hegemónica.

\section{Metodología}

Se realizó un estudio descriptivo con fines interpretativo y se utilizó una metodología cualitativa (Ruiz, 1996; Valles, 2000; Salinas y Cárdenas, 2008). Se buscó comprender los discursos en un sentido global. Se realizaron largas jornadas de observación, entrevistas informales y en profundidad a mujeres/garzonas, clientes y a dos dueños de salas de cerveza/schoperías.

\section{Procedimiento metodológico}

Se analizaron las interacciones de género entre clientes y grazonas en cuatro schoperías de Calama. Estos locales fueron escogidos a través de un proceso de observación in situ y de acuerdo a las sugerencias de informantes claves: que fueran schoperías muy frecuentadas en la ciudad, que tuvieran una antigüedad mínima de cinco años y que asistieran preferentemente trabajadores mineros. Más información respecto a las características de las salas de cerveza o schoperías se puede hallar en Barrientos y otros (2009).

Se realizaron largos períodos de observaciones, durante el 2007 y parte de 2008 (50 días en total, preferentemente fines de semana), lo que 
posibilitó a través de entrevistas informales, tomar contacto con los clientes, propietarios, garzonas y público en general. Además, se realizaron dos grupos de discusión con trabajadores mineros y tres con parejas de trabajadores. Se hicieron seis entrevistas formales en profundidad con garzonas. Además, durante los periodos de observación se mantuvieron diversas entrevistas informales con las mujeres, de acuerdo a las posibilidades que se daban durante el turno en la schopería. Todas las entrevistas fueron grabadas y transcritas exhaustivamente, previa autorización de los participantes. Las entrevistas en profundidad tuvieron una duración promedio de $90 \mathrm{mi}-$ nutos y se realizaron fuera del horario de trabajo. Los nombres de los (as) participantes fueron modificados, respetando el principio de confidencialidad de las investigaciones cualitativas.

\section{Análisis}

El análisis de discurso implicó que nos fijáramos en las palabras como modo de conocer la estructura social, y los procesos de significación y construcción del sentido (Murillo y Mena, 2006). Se buscó hacer un Análisis Crítico del Discurso (en adelante ACD), como una forma de abordar los problemas sociales que se van concadenando en ellos (exclusión, inequidad, entre otros). El ACD es una denominación genérica que se aplica a un planteamiento dedicado a estudiar el habla, el discurso y la comunicación (Van Dijk, 1997, Meyer, 2003). El ACD permite visualizar la relevancia social y política del texto, y muestra una dimensión de significado que sobrepasa el encasillamiento que conlleva un marco situacional concreto (Hennecke, 2004). Desde estas propuestas, realizamos una codificación del material recopilado en base a la Teoría Fundamentada (Strauss y Corbin, 2002), y con la aplicación que de ella hacen Trinidad y otros (2006). Debido a la codificación y segmentación de los discursos realizada, los segmentos de las entrevistas incluidos en el presente artículo, no se identifican los nombres ni las características de las entrevistadas, ya que hay una selección de los códigos más sustantivos de cada una de ellas, reduciendo al máximo el contexto discursivo.

\section{Contextualización de las salas de cerveza/schoperías}

En el norte de Chile, Calama se encuentra ubicada a 215 kilómetros de Antofagasta, con una población aproximada de 143.000 habitantes. En esta ciudad, las salas de cerveza/schoperías junto a otros locales del rubro constituyen uno de los negocios más rentables. En la década de los 80 ', se inicia la contratación de mujeres jóvenes y atractivas que provenían preferentemente del centro y sur del país. Con la inclusión de ellas, se concentró la entretención masculina, convirtiéndose en la actualidad en la principal forma de esparcimiento de los trabajadores mineros de la zona. En términos generales, las schoperías son espacios de consumo de cerveza. Actualmente, las mujeres/garzonas que allí trabajan son en su mayoría solteras. Las hay con y sin hijos y provienen de Perú, Bolivia y del noreste argentino, o de la zona sur del país, generalmente de la Región del Bio-Bío (Barrientos y otros, 2009). Sus edades fluctúan entre los 20 y 35 años de edad. El nivel educacional es varia- 
ble, algunas tienen la enseñanza media incompleta, otras cuarto año medio rendido, y las menos, son estudiantes universitarias.

\section{Resultados}

A continuación el cuadro sintetiza el proceso de categorización de los datos. En ellos se exponen los distintos ámbitos que aborda la discriminación de género y modos relacionales que identificaron en los discursos de las participantes.

\section{Cuadro N 1 Categorías Centrales}

\begin{tabular}{|c|c|c|c|}
\hline Subtantivos & Conceptuales & $\begin{array}{c}\text { Proceso social } \\
\text { básico }\end{array}$ & Categoria central \\
\hline 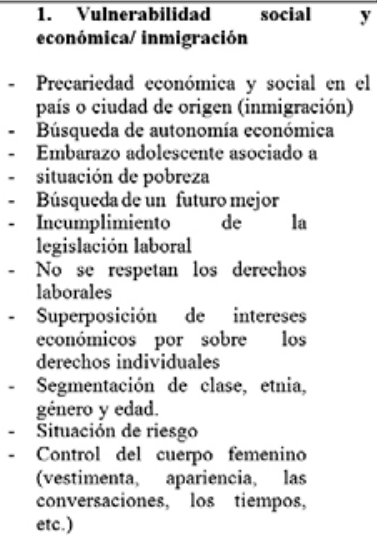 & $\begin{array}{l}\text { El trabajo como } \\
\text { enclave de } \\
\text { discriminación } \\
\text { e inequidad de } \\
\text { género }\end{array}$ & Incertidumbre & $\begin{array}{c}\text { Empoderamiento } \\
\text { Femenino }\end{array}$ \\
\hline $\begin{array}{l}\text { 2. La construcción del sujeto } \\
\text { femenino / } \\
\text { Imaginario asociado a las schopería y } \\
\text { a Calama } \\
\text { - Garzonas como prostitutas } \\
\text { - Les interesa el dinero } \\
\text { - Buscan marido } \\
\text { - Son fáciles } \\
\text { - El valor del cuerpo (como mercancia) } \\
\text { - Estigmatización } \\
\text { - Acoso sexual y psicológico } \\
\text { - Prestigioy estatus de los trabajadores de } \\
\text { CODELCO } \\
\text { - Sitios donde se ejerce la prostitución } \\
\text { - Negocio lucrativo } \\
\text { - Consumo excesivo de alcohol } \\
\text { - La actividad económica facilita el } \\
\text { comercio sexual, el alto consumo de } \\
\text { alcohol y drogas } \\
\text { - Alto crecimiento económico asociado a } \\
\text { la actividad minera } \\
\text { - Alto endeudamiento } \\
\text { - Centro de atracción laboral } \\
\text { - Alto consumo } \\
\text { - Problemas familiares } \\
\text { - Presencia de trabajadoras inmigrantes de } \\
\text { - Alto costo de la vida en la ciudad } \\
\text { (servicios básicos, arriendo, entre otros.) }\end{array}$ & $\begin{array}{l}\text { El control } \\
\text { sobre las } \\
\text { garzonas } \\
\text { como } \\
\text { estrategia } \\
\text { des- } \\
\text { estigmatizac } \\
\text { ión de las } \\
\text { Schoperia } \\
\text { Crecimiento } \\
\text { económico } \\
\text { versus } \\
\text { precariedad } \\
\text { social }\end{array}$ & $\begin{array}{c}\text { Disciplinamiento } \\
\text { de las mujeres }\end{array}$ & Mandatos de género \\
\hline
\end{tabular}




\section{Vulnerabilidad social y económica e inmigración}

Las mujeres que trabajan como garzonas en las schoperías de Calama provienen de los países limítrofes principalmente y de la zona sur de Chile. Esta situación perfila la creciente migración que se ha producido en la región en estos últimos años. La mayoría de los inmigrantes son atraídos por las positivas expectativas de crecimiento económico de la región. Cabe hacer notar que en caso de los extranjeros, algunas de las razones asociadas a este fenómeno, son la disparidad económica y social entre los países, las condiciones de pobreza, la falta de oportunidades y la búsqueda de perspectivas de mejor vida (CEPAL, 2006). El Censo de 2002 da cuenta de lo anterior, ya que en Chile existen 195.320 extranjeros residentes, de los cuales 50.448 son argentinos, 39.084 peruanos, 11.649 bolivianos y 9.762 españoles. De hecho, el flujo migratorio en el país se ha incrementado en un 75\% desde 1992 al 2002 (Ibid, 2006).

Junto al aumento de la inmigración, se advierte un incremento constante de la feminización de este fenómeno (Cárdenas y otros, 2010). El caso de la comunidad peruana es una evidencia clara de dicho fenómeno, pues un 63\% corresponde a mujeres (Martínez, 2003). Un estudio reciente realizado el 2008, sobre las mujeres extranjeras que ejercen el comercio sexual en la Región de Antofagasta, destaca la proporción de peruanas con un $27,5 \%$ colombianas $23,5 \%$ y ecuatorianas con $18,6 \%$, y visualiza la inmigración como un fenómeno importante asociado al comercio sexual y a la discriminación (Silva y otros, 2008).

Coincidente con los datos expuestos, las entrevistadas, efectivamente, han llegado al norte del país motivadas por superar los problemas económicos y/o familiares que las aquejan en sus países o ciudades de origen. La mayoría de ellas son jóvenes madres solteras sin pareja, por lo tanto, lograr la mantención de sus hijos es una preocupación central para ellas. Además, que no tengan pareja, facilita la decisión de inmigrar en búsqueda de mejores perspectivas laborales. Consecuentemente, la situación de premura económica que las aqueja, se expresa en que muchas de estas mujeres se encuentran en una condición inicial de vulnerabilidad, por lo tanto, bajo estas circunstancias las salas de cerveza /schoperías y otros locales del rubro son negocios lucrativos en la zona minera en los que se ofrecen las oportunidades laborales "más rentables” para este sector de la población (mujeres jóvenes).

Esta condición de vulnerabilidad social y económica connota la llegada de las mujeres a la zona y la presión por aceptar las precarias condiciones laborales que se les ofrecen, ya que pese a las atractivas remuneraciones, no tienen contrato de trabajo, el empleo es temporal, no tienen cobertura previsional, y en el caso de las extranjeras, se suman las dificultades para obtener visa de trabajo, entre otras. Por ejemplo, algunas entrevistadas mencionan:

"los arriendos son caros”, "la ciudad es muy sucia”, “en la noche es peligrosa”, "hay travestis en las esquinas", "venir de la selva y lle- 


\section{gar a un desierto es difícil” (garzonas schoperias).}

Este panorama configura el proceso de discriminación al que se ven afectadas, evidenciándose que condiciones como la edad de las mujeres, el género, la raza y/o clase social se relacionan, de una u otra manera, gatillando este fenómeno. En otras palabras, las garzonas son discriminadas, ya sea porque son mujeres y jóvenes, mujeres y madres solteras, mujeres negras o con rasgos indígenas y/ o mujeres en situación de pobreza. Respecto a ello, algunas mujeres garzonas de schoperias señalan:

"muchas mujeres piensan que las garzonas son "prostitutas", "si tomas un café con alguien, estás engañando al mino", "es mejor no contar que trabajas en una schopería por el mal prestigio que tienen”, "las colombianas son vistas como "prostitutas", "si te ríes estás puteando", "hay acoso de los clientes”, “cuando llegué quería llorar, no quería atender mesas", "los dueños te someten a muchas cosas", "hacen y deshacen con las niñas".

A esto se agrega, el estigma que existe sobre los propios locales, como sitios asociados al comercio sexual (sucios, oscuros, hay prostitución). Respecto a esto, las garzonas expresan que no hay comercio sexual, aunque, si alguna de ellas establece relaciones sexuales y afectivas con los clientes, éstas son fuera de los locales y después del horario laboral.

Estas trabajadoras son ubicadas por los propietarios de las schoperías a través de contactos personales o de agencias de empleo. Los dueños las seleccionan de acuerdo al gusto de los clientes. Respecto a esto, algunos propietarios señalan que:

"Puede ser una chiquita, puede ser una gordita, pero tiene que tener ángel, les "mirai" las piernas si soy fea no vay a trabajar, su carita flaquita, su buen poto, su buena teta", "llegan a través de agencias", "a través del contacto de una amiga”.

El relato anterior pone de manifiesto que lo que importa es mantener el interés de los clientes por asistir a la schopería, ya que el negocio se sostiene por el consumo de alcohol de los trabajadores mineros fundamentalmente y en el buen servicio de las chicas que en estos locales trabajan. Asi, un cliente afirma que:

“Aquí el producto es uno solo las mujeres que atienden y si no, no hay novedad”)

Las motivaciones que tienen las mujeres para trabajar en las schoperías se relacionan con el interés que tienen por alcanzar autonomía económica y juntar dinero durante un tiempo para concluir estudios o ayudar a la familia. Estas necesidades soslayan, en alguna medida, el rechazo o incomodidad que experimentan al comienzo cuando se inician en esta actividad laboral, ya sea porque deben usar ropa ajustada, faldas cortas, 
taco altos, porque no pueden sentarse con los clientes o porque deben trabajar hasta altas horas de la noches, es decir, deben adaptarse a la performance masculina que predomina en estos espacios y lidiar con el acoso de los hombres. Respecto a ello, algunas entrevistadas afirman que:

“Me sentía perseguida, me miraban”, "la vestimenta es muy exagerada”, “aguantar a los curados (borrachos)”, “trabajar en calzonetas”, "se muestra mucho el cuerpo”, “malestar por el uniforme”, "trabajar hasta tarde”, "no poder sentarte”.

Las garzonas evalúan este trabajo como transitorio y piensan que futuro para ellas será mejor e, incluso, algunas ven esta labor en las schoperías como un primer paso, para luego acceder a un empleo en alguna empresa minera de la zona. Por lo mismo, ellas logran empoderarse en este entorno, sobreponiéndose a la condición inicial de vulnerabilidad, puesto que aprenden los códigos interaccionales de estos espacios: controlar las relaciones con los clientes en la schopería, manejar la seducción mediante conversaciones, diálogos, miradas y movimientos entre las mesas sirviendo a los hombres. La mayoría de los clientes se encuentra, avanzada la jornada, más frágiles producto del excesivo consumo de alcohol y, por tanto, allí son ellas las que dominan el escenario, se apropian de los discurso y manejan la dinámica del local. Esto último es reconocido desde el interaccionismo como la capacidad de las personas de saber cómo actuar y cómo actuarán los demás, es decir, comparten significados preestablecidos acerca de lo que se espera en la acción de los participantes (Blumer, 1986, citado en Reynoso, 1998). Las mujeres logran control del espacio, conocen a los clientes y cómo deben actuar frente a ellos, en la medida que los hombres sucumben por el exceso de la cerveza. Allí son ellas son las que juegan con el deseo masculino, utilizando la seducción como estrategia de empoderamiento.

Sin embargo, este empoderamiento relativo que logran las garzonas frente a los clientes se manifiesta junto a la incertidumbre que ellas experimentan debido a la vulnerabilidad y discriminación que las afecta. Más aún, podemos precisar que este poder es lo que se conoce como un poder periférico o local y que se asocia al poder que ejercen las mujeres en el ámbito doméstico y que se diferencia del dominio global que ejercen los hombres en el cual ellas están subordinadas (Ramirez, 2006).

\section{La construcción del sujeto femenino imaginario asociado a las schopería y a Calama}

En otra dimensión, las garzonas experimentan la tensión que implica transgredir los mandatos de género tradicionales, es decir, no responder al imaginario de buenas madres, sumisas, discretas, atentas o invisibles. Por el contrario, como garzonas de schoperías, deben satisfacer los requerimientos de estos locales y la performance masculina, esto es como "objeto de deseo". En otras palabras, deben seducir, coquetear, mostrase, jugar con los clientes y facilitar el consumo de alcohol. En un primer momento, 
al iniciarse en este trabajo, las chicas se sienten inseguras, incomodas e, incluso, se resisten. Por ejemplo, algunas entrevistadas señalan que:

"Cuando llegué quería llorar”, "me sentía perseguida”, "malestar por el uniforme", "la vestimenta es muy exagerada, muy corta", "las colombianas son vistas como prostitutas", "no se puede fumar, ni tomar". "hablan de las chicas por atrás, dicen que son maracas", "hay acoso de los clientes".

Luego, cuando las mujeres logran un mayor dominio del entorno, comienza el empoderamiento de ellas, es decir, se sienten más seguras, despliegan estrategias que involucran la manipulación, el jugar. El cuerpo femenino como objeto, es sexualizado y es la principal estrategia de interacción de género; las mujeres coquetean y juegan con el deseo masculino. Ellas usan sus atributos físicos ante los hombres para lograr control del espacio, pero siempre en el marco de un sistema sexo/género patriarcal que se refuerza en este contexto productivo.

Las garzonas experimentan relaciones de contradicción consigo mismas ya que perciben el fuerza del estigma que circula respecto de ellas, entre el imaginario social que son "la prostituta" o "la dama". Ambos estereotipos se recrean y refuerzan en un proceso relacional que va marcando la identidad de las mujeres trabajadoras de las schoperías. Estos estereotipos tienen un frágil equilibrio en el imaginario de los clientes y de la ciudadanía en general. Por ejemplo, cuando los hombres desean invitar a alguna garzona para reunirse fuera del horario de trabajo, ellos utilizan todos los subterfugios de la seducción para hacerlas sentir una dama con expresiones como. Por ejemplo, algunas garzonas de schopería cuentan que se dice de ellas:

"Eres la más bella”, “me casaría contigo” o "nadie me espera en la cama”,"hay hombres "lachos" que te dejan 3.000 ó 5.000 pesos de propina", "muchos son casados con plata". .

Pero si la mujer rechaza la invitación, inmediatamente es tratada como "maraca", "que se cree”, es decir, el comentario, rumor o burla masculina se orienta a denigrarla. En consecuencia, ellas deben ser cuidadosas para evitar la reacción del hombre rechazado. Por ejemplo, algunas mujeres afirman que:

"Hay que negarse amablemente”, "si aceptan invitación dicen que las mujeres son "maracas" y si te niegas te tratan mal", "los clientes hacen propuestas sexuales", "los hombres miran como si no han visto a una mujer”.

A la complejidad de estas interacciones que se dan en la dinámica interna de las schoperías, se agregan los mandatos de género que son reforzados desde el exterior por los habitantes de la ciudad; allí se destaca el rol que juegan las "otras", vale decir, aquellas mujeres que son esposas y parejas, quienes difunden fácilmente rumores, estigmas y estereotipos sobre 
las garzonas de las schoperías. Las entrevistadas hablan de este fenómeno que se encuentra legitimado en la ciudad, y que ha sido, inclusive difundido en la prensa local y en los medios de comunicación nacional, fortaleciendo el estigma de Calama como "campamento minero"

Por ejemplo, algunas entrevistas señalan que:

"muchas mujeres piensan que las garzonas son maracas”, "que les interesa solo el dinero", "que buscan marido", "si tomas un café con alguien, estás engañando al mino”,"es mejor no contar que trabajas en una schopería, por el mal prestigio que tienen”.

De esta manera, se produce una interacción intragénero entre las garzonas y "las otras" (mujeres) la que, por una parte, desvaloriza a estas trabajadoras por representar la transgresión de los mandatos de género tradicionales (buena madre, abnegación, femineidad, discreción y entrega) y, por otra, estas mujeres ("las otras"), irrumpen como verdaderos baluartes de estos constructos tradicionales y que sostienen el sistema sexo/género hegemónico. Por lo tanto, la transgresión que representan las garzonas a estos valores, activa el control social, el disciplinamiento y el rechazo por parte de la ciudadanía en general ("los dueños hacen y deshacen con las niñas"). Lo anterior, da cuenta del juego de doble moral que se produce entre los distintos actores involucrados (trabajadores, garzonas, dueños de los locales, autoridades y público en general) y que, paradójicamente, sostiene el lucrativo negocio, por tantos años en la zona. Sin embargo, igualmente se han establecido, aunque menos relaciones más estables entre las garzonas y los clientes, pero no es lo habitual.

Por último, la selección de las garzonas por parte de los dueños de éstos locales muestra, prácticas discriminatorias. Por ejemplo, la apariencia física constituye una condición para lograr el trabajo, es importante que las mujeres no tengan rasgos indígenas, su lugar de procedencia, si son peruanas o bolivianas, el color de la piel, ojala lo más blancas posibles, la contextura, si son o no voluptuosas, la forma de las piernas y edad, todos atributos relevantes para lograr o no el empleo. Asimismo, se evidencia la desvalorización del sujeto femenino y la centralidad del cuerpo. Incluso, las propias mujeres refuerzan estos estereotipos de género; reconocen entre ellas quienes son las preferidas o las que tienen más clientes o las que reciben mejores propinas, de acuerdo a las características mencionadas. Esta realidad facilita el conflicto entre ellas. Muchas veces son las mismas compañeras quienes inician comentarios negativos, e incluso, se discriminan entre aquellas que son nuevas en el trabajo y las más antiguas.

\section{Conclusiones}

El desarrollo de una investigación de este tipo nos abre una serie de nuevas interrogantes, puesto que acceder a los discursos de los actores in situ, es una oportunidad de reconstrucción teórica y metodológica, frente a 
una realidad compleja, en la que se entraman distintas problemáticas sociales, culturales, de género, entre otras. La minería así emerge como un laboratorio social, que requiere distintos abordajes, superando los enfoques tradicionales que se centran en su valor económico.

Aunque, la gran minería del cobre en la Región de Antofagasta es la principal actividad productiva del país, caracterizada por los altos niveles de modernización y la incorporación de tecnología. Este mismo contexto productivo favorece un sistema sexo/género hegemónico. En este sistema la masculinidad es reforzada de acuerdo a la capacidad del trabajador minero de ser proveedor y jefe de familia, lo que produce como efecto un reconocimiento social y la posibilidad de imponer su autoridad mediante el poder asociado al dinero y al lugar que ocupa en el espacio laboral, transformándose en un ascendiente sobre las mujeres y sobre otros hombres. Esta realidad se diferenciaría de los avances que se han experimentado en otras zonas del país (por ejemplo, Región Metropolitana) donde los estudios muestran una identidad masculina más diversificada.

En otras palabras, la alta concentración de las oportunidades laborales que ofrece la minería y las empresas asociadas al rubro, configuran un área laboral eminentemente masculina, y con ello el predominio de una identidad de género hegemónica. Por ende, las schoperías son sitios que recrean y refuerzan la hegemonía de género donde las mujeres se encuentran subordinadas al poder masculino.

En estos locales, los hombres se apropian del espacio social, hacen uso de éste y construyen territorios propicios para su propia performance. Todo lo que allí acontece tiene significados masculinos: beber, fumar, comer, charlar, acosar o seducir a las mujeres que allí trabajan. Los hombres compiten entre sí por la atención de las mujeres asi como rivalizan en otras áreas, por ejemplo, en el trabajo. Aquí ellos despliegan su masculinidad, lo que se traduce en un dominio de la escena mediante gestos, ademanes, risas, garabatos o coqueteos. Finalmente, expresan y ejercen su poder mediante el dinero.

La discriminación femenina en las schoperías se expresa en distintas ámbitos. Por ejemplo, no se cumplen los derechos laborales ya que se superpone el interés económico por sobre la dignidad de las mujeres; las trabajadoras son discriminadas de acuerdo a las características físicas al momento de ser contratadas (color de la piel, apariencia, entre otras), y hay una permanente tensión entre el estigma de las garzonas (que son fáciles o que buscan marido) y lo que se espera de ellas como mujeres (buenas madres, esposas y abnegación). Por consiguiente, los constructos de género se activan como mandatos una y otra vez en el imaginario colectivo de clientes, garzonas y de la ciudadanía en general.

Además, pese a los niveles de empoderamiento y autonomía que muchas de ellas desarrollan mantienen el deseo (ilusión) de conocer una pareja que les permita dejar el trabajo y resolver los problemas 
socioeconómicos o familiares que las aquejan, lo que es un antecedente más de la vigencia de los mandatos de género tradicionales en la configuración de su propia identidad como sujetos femeninos, donde persiste por ejemplo, la ilusión de conocer una figura masculina "salvadora”.

La precariedad del trabajo femenino y la discriminación son elementos constituyentes de la condición de estas mujeres, pese a que ellas reconocen percibir una mejor remuneración comparada a la que recibirían desempeñándose en otras áreas de servicio (por ejemplo, trabajadoras de casa particular, vendedoras o promotoras). La discriminación apunta no sólo a condiciones materiales precarias como extensas jornadas, ausencia de contrato, incumplimiento de acuerdos laborales, sino también a aspectos más simbólicos como la mala reputación y los rumores que se recrean sobre ellas, delineando un proceso identitario contradictorio y en permanente construcción.

Las interacciones (modelos relacionales) de género que se establecen en estos locales se encuentran asociadas a una serie de códigos verbales y no verbales y a representaciones que en general resaltan el cuerpo femenino como objeto sexualizado. Las mujeres son conscientes de sus atributos y los efectos que provocan en los hombres, más aún si ellos se encuentran bajo la influencia del alcohol, por lo tanto, ellas logran un cierto control del espacio, competencia vital para la sobrevivencia en dichos espacios. Aunque, esta dinámica refuerza el sistema sexo/género hegemónico, ya que no hay una valoración del sujeto mujer en igualdad de condiciones, son ellas las que están al servicio del deseo masculino. Ellas aprenden los códigos interaccionales que se gatillan en estos ambientes, tienen control sobre sus movimientos, interacciones, conversaciones, miradas, sobre sus respuestas de aceptación o rechazo frente a las insinuaciones de los hombres. Pero, a la vez, sienten el peso del estigma y la discriminación por trabajar en estos sitios. Las mujeres, en las schoperías usan la seducción y su cuerpo, como decíamos, como principal estrategia para incentivar, mediar o aplacar el acoso o deseo masculino.

A esto se agrega el estigma asociado a las mismas schoperías (sitios sucios y oscuros, prostitución, entre otros) y a la actividad que allí verdaderamente acontece (beber cerveza, sitios de hombres, chicas jóvenes que atienden, consumo de cigarros, compañía, conversación), es decir, hay una visión de estos locales que no se relaciona directamente con la dinámica e interacciones que allí se generan.

Calama refleja como quizás ninguna otra ciudad del país, los graves problemas de centralización, mala gestión e inequidad, ya que a la aridez del desierto, se suma el aislamiento geográfico, la precariedad urbana, las temperaturas extremas, los altos índices de contaminación y el alto costo de la vida para los habitantes, lo que facilita, entre otras cosas, la alta concentración de locales que expenden bebidas alcohólicas y el comercio sexual. En consecuencia, las condiciones de vida que se generan en la zona delatan, por una parte, la precariedad laboral, social y cultural y el tipo de 
interacciones de género que allí se recrean. Y, por otra, se grafica una disociación real y simbólica con los cambios culturales, sociales y económicos que han tenido las mujeres en el país y que han posibilitado un mejor y mayor posicionamiento de éstas, como sujetos de derecho. Así, la gran minería del cobre yergue como uno de los centros productivos más modernos del país y, a la vez, con altos niveles de rezago en lo referido a mayor equidad de género en los distintos ámbitos de la vida social (trabajo, relaciones interpersonales y roles familiares, entre otros).

Por último, en este escenario productivo se favorece la reproducción de enormes brechas entre los avances tecnológicos y las oportunidades laborales masculinas, versus las condiciones laborales que deben sobrellevar las mujeres y sus oportunidades. Esto último gráfica el sentido inverso que tiene el desarrollo en la región respecto a las políticas de igualdad de oportunidades que se están promoviendo a nivel nacional e internacional en los distintos ámbitos de la sociedad. Por lo tanto, se requieren políticas a nivel público y privado, con un fuerte acento local, que promuevan la equidad de género en forma transversal, entendiendo que sus efectos trascienden al crecimiento económico, social y cultural de la región. 


\section{Nota}

${ }^{*}$ Este trabajo ha sido posible gracias al financiamiento del proyecto Fondecyt $\mathrm{N}^{\circ} 1070528$. Agradecemos el trabajo en terreno de Pablo Rojas Varas. Y los valiosos testimonios de mujeres y hombres de Calama que accedieron a participar en este proceso investigativo, como asimismo a cada uno de los evaluadores. 


\section{Bibliografía}

Abramo, L. (2004), “¿Inserción laboral de las Mujeres en América latina: una Fuerza de trabajo secundaria? "Revista Estudios Feministas, 12(2), 224-235, Universidad Federal de Santa Catarina, Florianópolis.

Andreu, J.; García, A. y Pérez, A. (2007), Evolución de la Teoría Fundamentada como Técnica de Análisis Cualitativo. Centro de Investigaciones Sociológicas, Madrid.

Aylwin, M.; Bascuñan, C.; Correa S.; Gazmuri, C.; Serrano, S. y Tagle, M. (1998), Chile en el siglo XX. Octava edición (6ta. en Planeta), Planeta, Santiago de Chile.

Barrientos, J.; Salinas, P.; Rojas, P. y Meza, P. (2009), “Minería, género y cultura. Una aproximación etnográfica a espacios de esparcimiento y diversión masculina en el norte de chile”. AIBR- Revista de Antropología Iberoamericana, 4(3): 385-408, España.

Bonder, G. (1999), “Género y Subjetividad: avatares de una relación no evidente”. En Género y Epistemología. Mujeres y Disciplinas, editado por S. Montecino y A. Obach. LOM ediciones, pp. 29-55, Santiago de Chile

Bourdieu, P. (2007), La dominación masculina. Anagrama Colección, España.

Cademartori, J. (2009), El Desarrollo Económico y Social de la Región de Antofagasta (Chile): Historia y Perspectiva. Universidad Católica del Norte, Editorial Ordhum, Antofagasta, Chile.

Carabi, A. y Armengol, J. (2008), La masculinidad a debate. Icaria Mujeres y Cultura, Barcelona.

Carbonero, M. y Levín, S. (Compiladoras) (2007), Entre familia y trabajo. Relaciones, conflictos y políticas de género en Europa y América Latina. Homo Sapiens Ediciones, Argentina.

Cárdenas, M.; Yañez, S.; Gómez, F. y Méndez, L. (2010), Estudio: Inmigración internacional en la región de Antofagasta. Opinión de la población residente en las ciudades de Antofagasta, Calama y San Pedro de Atacama sobre la inmigración en la región. Documento de Trabajo Número 1, marzo de 2010. Universidad Católica del Norte - Facultad de Humanidades Escuela de Psicología Centro de Atención Psicosocial a Inmigrantes Ignacio Martín-Baró.

Careaga, G. y Cruza, S. (2006), Debates sobre Masculinidad. Poder, Desarrollo, Políticas Públicas y Ciudadanía. Universidad Nacional Autónoma de México, México. 
Carmagnani, M. (2006), El salario minero en Chile colonial, su desarrollo en una sociedad provincial: El norte chico 1690-1800. Dirección de bibliotecas, archivos y museos, Santiago de Chile.

CASEN (2006), Encuesta de Caracterización Socioeconómica Nacional [en línea]. Disponible en www.mideplan.cl

CEPAL (2008), Comisión Económica para América Latina, Anuario Estadístico de América Latina y el Caribe, consultado el 9-6-09 [en línea] Disponible en: http://websie.eclac.cl/anuario_estadistico/anuario_2008/docs/ ANUARIO2008.pdf

CEPAL (2008), Migración internacional, derechos humanos y desarrollo en América latina y el Caribe. www.acnur.org/biblioteca/pdf/4089.pdf (Recuperado el 26 Noviembre de 2008).

Connell, R. (2003), Masculinidades. PUEG. Universidad Nacional Autónoma de México. Programa Universitario de Estudios de Género, México.

Connell, R. (2006), “Desarrollo, Globalización y Masculinidades”. En. Debates sobre Masculinidades. Poder, Desarrollo, Políticas Públicas y Ciudadanía. G. Careaga y S. Cruz Sierra (Eds.), Universidad Autónoma de México p.185-210, México.

CONSEJO MINERO (2003), Informe de la Gran Minería Chilena. Editorial Trineo, [en línea] Disponible en: www.consejominero.cl/home/doc/ 26informe2002.pdf

Cortés, J. (2004), Hombres de Mármol. Códigos de representación y estrategias de poder de la masculinidad, Editorial EGALES, Barcelona.

Dunbar, M.T. (1994), Going for gold: men, mines and migration. Witwatersrand University Press, Johannesburg.

ENCLA (2009), Resultados de la Sexta Encuesta Laboral, Noviembre 2009, Documento Producido por la Dirección del Trabajo, [en línea] Disponible en: www.direcciondeltrabajo.cl

GIM (Grupo de Iniciativa Mujeres) (2002), El Nuevo Contrato Social Dirigido a las Mujeres: balance de una década de democracia en Chile. GIM, Cedem, Cem, Corporación La Morada, Corsaps, Domos, Flacso, Fundación Instituto de la Mujer, Isis Internacional, Mench, Prosam, Santiago de Chile.

Gutiérrez, M. (compiladora) (2007), Género, Familia y Trabajo: Rupturas y continuidades. Desafíos para la investigación política, CLACSO, Buenos Aires.

Hennecke, A. (2004), Diskurs als Fluss von Wissen duch die Zeit. Reflexionen über das Verhältnis zwischen Diskurs und Wirklichkeit. Co- 
municarte editorial, Argentina.

Instituto Nacional de Estadísticas (INE), (2007), Estadísticas de Trabajo y Previsión [en línea]. Disponible en www.ine.cl

Innova Minería Antofagasta (2008), [en línea]. Disponible en: http://www.innovamineriaantofagasta.cl/contenidos.phtml?seccion=35

Joas, H. (1998), “Interaccionismo Simbólico”. En La Teoría Social Hoy. Anthony Giddens, Jonathan Turner y Otros, Editorial Alianza Universidad, pp. 112-154, Madrid.

Joseph, I. (1999), Erving Goffman y la Microsociología. Gedisa Editorial, pp.125, Madrid.

Klubock, Thomas (1995), "Hombres y mujeres en el teniente, la construcción de género y clase en la minería chilena del cobre 1904-1951”. [Artículo]. En Godoy, L. [et al.], Disciplina y desacato. Construcción de identidad en Chile, siglos XIX y XX. Coedición SUR/CEDEM. Pp. 110-123, Santiago de Chile.

Lamas, M. (2002), Cuerpo: Diferencia Sexual y Género. Taurus Pensamiento, México.

Lardé. J, Chaparro. E, Parra. C. (2008), El aporte del sector minero al desarrollo humano en Chile: El caso de la región de Antofagasta. Publicaciones Cepal, Santiago de Chile.

Macheke, C. y CampbelL, C. (1998), "Perceptions of HIV/AIDS on a Johannesburg gold mine”. South African Journal of Psychology, 28(3), 146-154.

Maigret, E. (2003), Sociología de la comunicación y de los medios. Fondo de la Cultura Económica, Colombia.

Martinez, G. (2007), “La construcción imaginaria de la sexualidad y la violencia masculina”. En. R. Montesinos (coord.), Perfiles de la Masculinidad, Universidad Autónoma Metropolitana. Pp. 47-73, México.

Martínez, J. (2003), Breve Examen de la Inmigración en Chile según los Datos Generales del Censo 2002. Documento de Trabajo №3. Organización Internacional para las Migraciones, OIM-Chile. www.bcn.cl/ carpeta_temas/temas_portada.2006-05-16.1264867506/PDF/ trabajo_cimal_03.pdf (Recuperado el 26 Noviembre de 2008).

Meyer, M. (2003), “Entre la teoría, el método y las políticas: la ubicación de los enfoques relacionados con el ACD”. En Métodos de análisis crítico del discurso, Ruth Wodak y Michael Meyer, Gedisa. pp. 35-59, Madrid. 
Montecinos, S. (1998), Análisis de impacto psicosocial, sistema de trabajo por turnos en la unidad familiar. Proyecto aprobado por el Consejo Regional de Antofagasta. Fondo de igualdad de oportunidades para las mujeres de la II Región. F.N.D.R. unidad técnica: Sernam II región. Ejecutado por Universidad de Chile, Facultad de Ciencias Sociales, Programa Interdisciplinario de Estudios de Género.

Montesinos, R. (2002), Las Rutas de la Masculinidad. Ensayos sobre el Cambio Cultural y el Mundo Moderno. Gedisa, España.

Montesinos, R., (2007), Perfiles de la Masculinidad. Universidad Autónoma Metropolitana, México.

Murillo, S. y Mena, L. (2006), Detectives y camaleones: el grupo de discusión. Una propuesta para la investigación cualitativa. Talasa Ediciones, Madrid.

OIT, Organización Internacional del Trabajo (2007), Tasa de participación de las mujeres [en línea]. Disponible en: http://www.oitchile.cl/pdf/ chile-mujer08.pdf

Olavarría, J. (2000), “De la Identidad a la Política: Masculinidades y Política Públicas: Auge y Ocaso de la Familia Nuclear Patriarcales en el Siglo XX”. En J. Olavarría y R. Parrini (Eds.), Hombres: Identidad/es y Violencia. Encuentro de Estudios de Masculinidades: Identidades, cuerpos, violencia y políticas públicas FLACSO- Chile, Universidad Academia de Humanismo Cristiano/Red de Masculinidades, pp 11-28, Santiago.

Olavarría, J. (2006), “Hombres e Identidad de Género: Algunos elementos sobre los recursos de poder y violencia masculina”. En Debate sobre Masculinidad, editado por Gloria Careaga, Salvador Cruz Sierra. Universidad Autónoma de México, pp. 115-130, México.

Otero, E. (2006), Comunicación Social, Editorial Universitaria, Santiago de Chile.

Quintero, A. (2007), Diccionario Especializado en Familia y Género. Editorial Hvmanitas de Editorial y Distribuidora Lumen, Argentina.

Ramírez, J. (2006), “¿Y eso de la masculinidad?: Apuntes para una discusión”. En. Debates sobre Masculinidad. Poder. Desarrollo. Políticas Públicas y Ciudadanía, editado por Gloria Careaga y Salvador Cruz. México: Universidad Nacional Autónoma de México, pp. 31-56.

Ramírez, J. y Silva, I. (2008), Globalización y desarrollo regional: evolución económica de las regiones chilenas, 1990-2002. Revista de CEPAL, 95, 103-124.

Reynoso, C. (1998), Corrientes en antropología contemporánea. Edito- 
Polis, Revista de la Universidad Bolivariana, Volumen 10, $N^{\circ}$ 29, 2011

rial Biblos, pp. 351, Buenos Aires.

Ruitenbeek, H. (1967), El mito del machismo. Paidós, Buenos Aires.

Ruiz, J. (1996), Metodología de la Investigación Cualitativa. Universidad de Deusto, Bilbao.

Ruiz, O., Solano, S. y Zapata, C. (1998), “Redes de mujeres pobladoras de la comuna de San Joaquín. Participación y ciudadanía emergente”. Proposiciones N28, Sur Ediciones, Santiago de Chile

Sagredo, R. y Gazmuri, C. (dirs.) (2005), Historia de la vida privada en Chile, Tomo I, El Chile Tradicional, de la Conquista a 1840, Aguilar Chilena Ediciones-Taurus, Santiago de Chile.

Salazar, G. y Pinto, J. (1999), Historia contemporánea de Chile II, actores, identidad y movimiento. LOM, ediciones, Santiago de Chile.

Salinas, P. (2002), “Ciudadanía y género. Mujeres y liderazgo en las provincias de Antofagasta, El Loa y Tocopilla”. Revista Electrónica de Trabajo Social, UDEC, Concepción.

Ídem (2007), "Los discursos masculinos como dispositivos de control y tensión en la configuración del liderazgo y empoderamiento femenino”. Revista de Estudios Feministas del Centro de Filosofía y Ciencias Humanas, 15(3), 541-562, Universidad Federal de Santa Catarina, Florianópolis.

Salinas, P. y Arancibia, S. (2006), “Discursos Masculinos sobre el Poder de las Mujeres en Chile. Sujetos y Subjetividades”. Revista Última Década. CIDPA, 14, 25, pp. 65-90, Valparaíso, Chile.

Salinas, P. y Cárdenas, M. (2009), Métodos de Investigación Social. Antofagasta, INTIYAN, Ediciones CIESPAL, Ecuador.

Sandin, M. P. (2003), Investigación Cualitativa en Educación. Fundamentos y Tradiciones. Mc. Graw Hill, pp. 258, Madrid.

Seidler, V. (2006), Masculinidades. Culturas globales y vidas íntimas. Montesinos Ensayo, pp. 262, España.

Servicio Nacional de la Mujer (2009), Agenda regional de género región de Antofagasta 2008-2010. [ en línea]. Disponible en: http://www.sernam.cl/ sistema_gt/sitio/integracion/sistema/archivos/file/pdf/generoantofagasta.pdf

Silva, J. (2008), Tacones Cercanos. Un estudio con perspectiva de género. Situación de las mujeres en el comercio sexual, Región de Antofagasta. Investigación financiada por el FNDR-código BIP N ${ }^{a}$ 30066633-0. SERNAM, Antofagasta. 
Strauss, A. y Corbin, J. (2002), Bases de la Investigación Cualitativa. Técnicas y procedimientos para desarrollar la teoría fundamentada. Editorial Universidad de Antioquia, Medellín.

Tallichet, S. (1995), "Gendered relations in the mines and the division of labor underground”. Gender \& Society, 9(6), 697-711.

Trautner, M. (2005), “Doing gender, doing class: the perfomance of sexuality in exotic dance club”. Gender \& Society, 19(6), 771-788.

Trinidad, A.; Carrero, V. y Soriano, R. (2006), Teoría Fundamentada "Grounded Theory”. La construcción de la teoría a través del análisis interpretacional. Centro de Investigaciones Sociológicas, Madrid.

Valdes, T. y Weinstein, M. (1993), Mujeres que sueñan: Las organizaciones de pobladoras 1973-1989, FLACSO, Santiago de Chile.

Valdés, T. y Olavarría, J. (1997), Masculinidad/es poder y crisis. ISIS Internacional/FLACSO, Santiago, Chile.

Valdés, T. (coordinadora) (2001), El indice de compromiso cumplido ICC. Una estrategia para el control ciudadano de equidad de género, FLACSO, Santiago, Chile.

Valcuende, J. y Blanco, J. (2003), Hombres La construcción cultural de las Masculinidades. Talasa ediciones. Serie Arcoiris, Madrid.

Valles, M. (2000), Técnicas cualitativas de investigación social. Reflexiones metodológicas y práctica profesional. Síntesis, Madrid.

Van Dijk, T. (1997), Racismo y Análisis Crítico de los Medios. Editorial Paidós, Barcelona.

Vergara. A. (2004), “Conflicto y Modernización en la Gran Minería del Cobre (1950-1970)”. Revista de Historia del Instituto de Historia de la Pontificia Universidad Católica de Chile, 37(2), 419-436.

Ídem ( 2007), “Ciudades privadas. La vida de los trabajadores del cobre”. En R, Sagredo. C, Gazmuri, (Eds.), Historia de la vida privada en Chile. El Chile contemporáneo. De 1925 a nuestros días, Taurus-Aguilar, pp. 84103, Santiago de Chile.

Viveros, M. (2001), Contemporary Latin American perspectives on masculinity. Men and masculinities, 3, 3: 237-260.

West, R. y Turner, L. (2004), Teoría de la Comunicación. Análisis y aplicación. Editorial Mc. Graw Hill, Madrid.

West, C. y Zimmerman, D. (1999), “Haciendo Género”. En. M. Navarro, 
Polis, Revista de la Universidad Bolivariana, Volumen 10, $N^{\circ}$ 29, 2011

C. \& R. Stimpson (compiladoras), Sexualidad, género y roles sexuales, Fondo de Cultura Económica. Pp. 109-143, México.

Zapata, F. (2002), Los mineros como actores sociales y políticos en Bolivia, Chile y Perú durante el siglo XX. [En línea]. [Consultado 15 de octubre, 2009) Disponible en: http://www.scielo.cl/scielo.php?pid=S071810432002002200006\&script=sci_arttext.

Recibido: 28.05.2010

Aprobado: 17.03.2011 


\section{Anexo \\ Códigos sustantivos y familias asociadas}

1. DATOS DE IDENTIFICACIÓN

Estudios

Edad

Lugar de nacimiento

Identificación de la familia

\section{LA SCHOPERÍA}

Motivación para trabajar en la Schopería

Para ayudar a la familia

Por dinero

Para independizarse

Pagan bien

Por necesidad

Para juntar dinero

Porque la mayoría son madres solteras

Como puente para luego entrar a una mina

Cómo llegan a trabajar a las Schoperías

A través agencia

A través del contacto de una amiga

Porque son extrajeras (peruanas, bolivianas)

Relaciones interpersonales en las Schoperías

Hay problemas con las mujeres

Hay chismes, chismes

Las mujeres hablan entre ellas

Hablan mal unas de otras

Hay conflictos, cagüines e intrigas

Las niñas antiguas tratan mal a las más nuevas

Deben pagar el derecho a piso y aguantar todo

Hay conflictos por los clientes

Si te ríes con alguno estás "puteando"

En lo positivo uno se apoya en las compañeras

\section{IMAGEN DE LAS MUJERES QUE TRABAJAN EN LAS SHOPERÍAS}

Muchas mujeres piensan que las garzonas son "maracas"

Si tomas un café con alguien, estás engañando al mino

Es mejor no contar que trabajas en una Schopería por el mal prestigio que tienen

\section{VISIÓN SOBRE CALAMA}

Los arriendos son caros 
La ciudad es muy sucia

En la noche es peligrosa

Hay travestis en la esquina es peligroso

Venir de la selva y llegar a un desierto es difícil

No quería volver a Calama

Uno se acostumbra a la ciudad

\section{VISIÓN SOBRE LA SCHOPERÍA}

Son vistas como prostíbulos

La gente piensa que somos "maracas" (prostitutas)

No me pareció malo

El prestigio de la Schopería es porque vienen trabajadores de Codelco, Gaby.

Son lugares fuertemente estigmatizados

\section{CÓMO SON LOS CLIENTES EN LA SCHOPERÍA}

Asisten hombres jóvenes, adultos, viejos, ancianos, todo tipo de hombre.

Hay acoso de los cliente

Asisten hombres que toman cerveza y no miran a nadie

Hay borrachos eufóricos y otros tranquilos

Cuando están borrachos te dicen cosas

Hay hombres que vienen a coquetear

Dicen que nadie los espera en la cama

Hay hombres "lachos", te dejan 3.000 o 5.000 pesos de propina

Los hombres escuchan música y se juntan con sus amigos

Muchos son casados con plata

Clientes que hacen propuestas sexuales

Los hombres miran como si no han visto a una mujer

Hablan de las chicas por detrás, dicen que son "maracas"

Si aceptan invitación dicen que las mujeres son "maracas" y si te niegas te tratan mal

Hay que negarse amablemente

Trabajar con tanto hombre es entretenido porque te piropean

\section{PROBLEMAS POR TRABAJAR EN LAS SCHOPERÍA}

Cuando llegué quería llorar, no quería atender mesa

Me sentía perseguida, me miraban

Malestar por el uniforme,

La vestimenta es muy exagerada, muy corta

Se muestra mucho (cuerpo)

Aguantar a los curados

Trabajar hasta tarde

No poder sentarte

Estar con tacos altos

Trabajar en calzonetas

Las colombianas son vistas como prostitutas 


\section{RELACIÓN DUEÑOS DE LA SCHOPERÍA}

Los dueños te someten a muchas cosas

No se puede sentar con un cliente

No se puede fumar, ni tomar

Hacen y deshacen con las niñas

En la piezas que te arrienda el dueño de la Schopería, no pueden entrar hombres

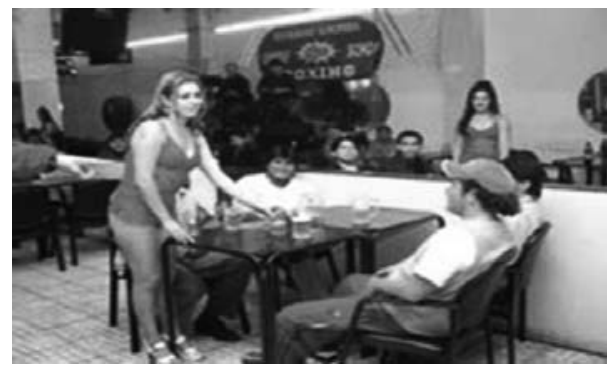

Sala de cerveza en Calama

\section{Provincia de Tocopilla, Antofagasta y El Loa}

Capital Regional: Antofagasta

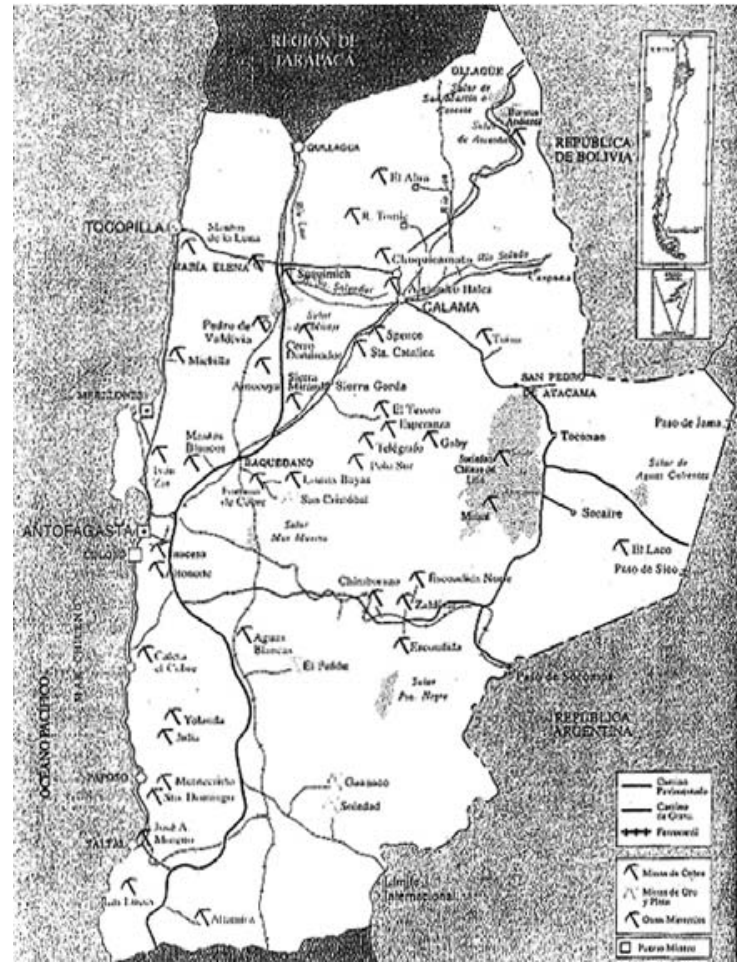

